\title{
Angiogenesis In Vitro Utilizing Murine Vascular Explants in Miniaturized 3-Dimensional Collagen Gels
}

\author{
May J. Reed ${ }^{\mathrm{a}}$, Mamatha Damodarasamy and Robert B. Vernon*,b \\ ${ }^{a}$ Department of Medicine, University of Washington, Harborview Medical Center, 325 Ninth Ave., Seattle, WA 98104, \\ USA; ${ }^{b}$ Hope Heart Matrix Biology Program, Benaroya Research Institute at Virginia Mason, 1201 Ninth Ave., Seattle, \\ WA 98101-2795, USA
}

\begin{abstract}
Models of angiogenesis in vitro are used to study blood vessel morphogenesis and the effects of compounds that influence vascular growth. Herein, we describe techniques to induce angiogenesis-like sprouting from explants of mouse aortae and microvessels cultured in 3-dimensional gels of native type I collagen. The gels are supported by rings of nylon mesh that are sized to fit in 96-well culture plates. This mechanically-supported, miniaturized, 3-dimensional culture system requires only small quantities of cells and reagents and facilitates handling, staining, and imaging by conventional and confocal microscopy.
\end{abstract}

Keywords: Mouse, fat, microvessels, aorta, explant, angiogenesis, 3-dimensional, collagen, gel, extracellular matrix, culture.

\section{BACKGROUND}

Angiogenesis, the generation of new vasculature from pre-existing blood vessels, is a critical phase of both normal and pathological processes such as wound healing and cancer. In vitro models of angiogenesis permit study of neovascularization and vascular morphogenesis, and also serve as assays to screen compounds for therapeutic regulation of blood vessel growth. The generation of blood vessel (i.e., capillary)-like structures in vitro occurs best in 3dimensional (3D) extracellular matrix (ECM) gels comprised of collagen, fibrin, or basement membrane material that simulate connective tissue environments in vivo. Typical models in vitro utilize either isolated endothelial cells (ECs) or vascular explants as starting material.

\subsection{Models Utilizing Isolated ECs}

ECM-based 3D models utilizing isolated ECs can generate tubular structures in vitro. The best of these, from a morphological perspective, uses human umbilical vein ECs (HUVECs) dispersed in type I collagen gels. In the presence of phorbol ester, the dispersed HUVECs rapidly develop into a network of thin-walled, multicellular tubes with wide, patent lumens that bear a striking resemblance to native capillaries [1]. Adult human microvascular ECs (HMECs), under specific conditions of stimulus, can also develop into patent tubes in 3D collagen gels [2]. ECM-based 3D models incorporating isolated, dispersed ECs are relatively easy to set up (given that purified ECs are available); however, such models have been criticized because they generate capillarylike tubes in a manner that is considered to be more representative of early embryonic vasculogenesis, rather than

\footnotetext{
*Address correspondence to this author at the Hope Heart Matrix Biology Program, Benaroya Research Institute at Virginia Mason, 1201 Ninth Ave. Seattle, WA 98101-2795, USA; Tel: 206-341-1349;

Fax: 206-341-1370; E-mail: rvernon@benaroyaresearch.org
}

true angiogenesis. The latter requires a "parent" blood vessel from which neovascular "sprouts" emanate. Nevertheless, the formation of networks of tubes from dispersed ECs may be accompanied by secondary, "angiogenesis-like" sprouting from the newly-formed tubes. HUVEC- and HMEC-based models have the advantage of utilizing cells of human origin and, therefore, are considered to be particularly useful for screening therapeutic compounds. To maximize the efficiency of screening assays, these models can be adapted to the miniaturized, 96-well format described in the following sections.

As alternatives to models utilizing isolated, dispersed ECs, other approaches have been devised that concentrate isolated ECs into focal sources within 3D ECM gels. These foci, which take the form of cell aggregates [3] or cells grown on the surfaces of microcarriers [4], simulate a parent vessel insofar as they are compact sources of ECs from which multicellular sprouts can form and invade the surrounding ECM. Although these models can sometimes be problematic (for example, some EC types are difficult to aggregate and microcarriers individually support relatively small numbers of cells), they continue to be useful in studies of vascular growth within 3D ECM.

\subsection{Murine Macro- and Microvascular Explants}

3D, ECM gel-based models in vitro using isolated ECs have been supplemented by 3D models that utilize explanted vascular segments which serve as parent vessels for angiogenesis-like sprouting. The latter are comprised of segments of macro- or microvessels that contain an endothelium and associated supportive (mural) cells. Although originally developed using aortae and adipose microvessels from rats as starting material [5-14], the availability of powerful technologies for manipulating gene expression in mice, in conjunction with an increased use of mouse models for studies of aging, have engendered a strong interest in angiogenesis models based on murine vascular 
tissue [15-19]. Methods for preparing macro- (i.e., aortic) and microvascular explants from mice are described below in "Description of Methods".

Vascular explants require a 3D ECM in which to sprout in vitro. Available forms of ECM include basement membrane derivatives such as Matrigel ${ }^{\mathrm{TM}}$, fibrin gels, and native type I collagen extracted from bovine dermis (Vitrogen ${ }^{\mathrm{TM}}$ ) [20] or the skin and tail tendons of rats [21, 22]. Collagen can also be isolated from mice - use of mouse collagen is particularly advantageous if one desires an "allmouse" system in which the explanted vascular tissue and supportive ECM are matched syngeneically [23]. Although commercial sources of mouse collagen exist, it is currently more cost-effective for investigators to prepare their own extracts. A simple method for extraction of native type I collagen from mouse tail tendons is outlined below in "Description of Methods".

Regardless of the source of cells and supportive ECM for vascular cultures, it has become increasingly important to develop in vitro angiogenesis assays in miniature formats, in order to efficiently utilize cells and reagents which are expensive or in limited supply. In 1999, we developed an angiogenesis assay, based on isolated ECs, which utilized rings of nylon mesh to support lenticular collagen gels small enough to fit in a standard 96-well cell culture plate [3]. Subsequently, we have adapted this miniaturized approach for use with explanted murine aortic segments [18] and microvascular tissue. Methods used to produce similar cultures are described below.

\section{DESCRIPTION OF METHODS}

\subsection{Murine Microvascular Explants}

Typically, explanted microvasculature for angiogenesis assays is obtained from masses of adipose tissue, such as that surrounding the epididymis $[5,7,12,14,15,19]$. To isolate microvessels from murine epididymal adipose tissue, 6-10 fat pads from 3-5 male mice are pooled, gently cut with small dissecting scissors into cubes $1-2 \mathrm{~mm}$ on each side, and placed in a $50 \mathrm{ml}$ Erlenmeyer flask filled with $20 \mathrm{ml}$ of Dulbecco's Modified Eagle's Medium (DMEM) (Invitrogen) containing $2 \mathrm{mg} / \mathrm{ml}$ of fatty acid free, low endotoxin, cell culture tested bovine serum albumin and $2 \mathrm{mg} / \mathrm{ml}$ of type II collagenase (Sigma-Aldrich) pre-warmed to $37^{\circ} \mathrm{C}$. The flask, with the suspended fat, is agitated in a rotary incubator/shaker at $37^{\circ} \mathrm{C}$ and $120-160 \mathrm{rpm}$ until the fat forms a slurry (digestion requires approximately 30 minutes). Blood vessels are separated from adipocytes by centrifuging the mixture at $1000 \mathrm{x} \mathrm{g}$ for 5 minutes - the adipocytes float to the top while the vasculature is pelleted. The vascular pellet is washed by gentle resuspension in $8 \mathrm{ml}$ of DMEM supplemented with 10\% fetal bovine serum (FBS) and antibiotics (100 units/ml of penicillin and $100 \mu \mathrm{g} / \mathrm{ml}$ of streptomycin) followed by 3 minutes of centrifugation at $1000 \mathrm{x}$. Washing is performed twice. The pellet is then resuspended in $8 \mathrm{ml}$ of DMEM/10\% FBS/antibiotics and poured through a $20 \mathrm{ml}$ syringe body fitted with a $25 \mathrm{~mm}$ diameter Swinnex ${ }^{\circledR}$ filter holder containing a $300 \mu \mathrm{m}$ mesh nylon screen, in order to remove tissue fragments and pieces of large-caliber vasculature. The filtrate, consisting primarily of microvascular segments and single cells, is then passed through a $20 \mathrm{ml}$ syringe body fitted with a $25 \mathrm{~mm}$ diameter
Swinnex holder containing a $30 \mu \mathrm{m}$ mesh nylon screen that traps the microvascular segments, but allows single cells to pass through. Subsequently, the microvascular segments are washed from the $30 \mu \mathrm{m}$ mesh screen into a $60 \mathrm{~mm}$ diameter tissue culture dish with jets of DMEM/10\% FBS/antibiotics delivered from a $10 \mathrm{ml}$ syringe fitted with an 18-gauge needle. Typical isolates consist of 20-40 microvascular segments with lengths of $30-150 \mu \mathrm{m}$. Within 30 minutes of isolation, the segments are suspended in 3D collagen gels, as described below.

\subsection{Murine Aortic Explants}

To obtain aortic segments from mice, the aorta is identified by its location with respect to the heart. This is more easily accomplished after careful removal of the lungs. The aorta is cut at the arch and at its junction with the diaphragm and transferred to a $60 \mathrm{~mm}$ diameter tissue culture dish containing $5 \mathrm{ml}$ of MCDB 131 medium (Invitrogen) supplemented with antibiotics. Isolated aortae are rinsed twice with this medium to remove extravasated blood and then are gently cleaned of loose perivascular connective tissue. A complete removal of this connective tissue minimizes fibroblast contamination of the explant. The cleaned aortae are cut transversely with a scalpel (fitted with a \#20 blade) into segments $1 \mathrm{~mm}$ in length. The cut is made by moving the curved edge of the scalpel blade through the aorta with a single rocking motion, which minimizes damage to the endothelial lining. Subsequently, the aortic segments are rinsed in MCDB 131 and, within 30 minutes, are placed into 3D collagen gels, as described below.

\subsection{Extraction of Collagen from Mouse Tails}

To prepare type I collagen extracts, tendons are peeled from the skinned tails of 3-5 mice by pulling the tendons away from the vertebral bodies with a strong tweezers or hemostat while cutting the tendons' connections to the vertebrae with a scalpel (fitted with a \#10 blade). At least 4 mice should be used if the animals are greater than 18 months of age. The isolated tendons are pooled, minced with small dissecting scissors, and immersed sequentially in 50 $\mathrm{mM}$ Tris-buffered saline (TBS) $(\mathrm{pH} \mathrm{7.4)}$, acetone, and 70\% isopropanol ( 5 minutes for each immersion). The collagen is extracted by stirring the tendons overnight at $4^{\circ} \mathrm{C}$ in $50 \mathrm{ml}$ of $0.05 \mathrm{~N}$ acetic acid. The resulting viscous solution is centrifuged at $4000 \mathrm{x} \mathrm{g}$ for 15 minutes at $4{ }^{\circ} \mathrm{C}$ to remove insoluble material. Collagen content (which is typically between 1.5 and $2.5 \mathrm{mg} / \mathrm{ml}$ ) can be quantified by the SircolTM Soluble Collagen Assay (Biocolor), which is specific for collagen and does not detect proteoglycans, elastin, or other ECM components. Collagen extracts should be refrigerated for storage, but never frozen.

\subsection{Preparation of Collagen Solution for 3D Gels}

Acidic extracts of mouse tail tendons and similar commercial extracts made from rat tail tendons or bovine dermis are comprised primarily of native collagen monomers. Adjustment of these extracts to physiological ionic strength and $\mathrm{pH}$, followed by heating to $37^{\circ} \mathrm{C}$, causes the monomers to assemble spontaneously into long, banded fibrils $70-100 \mathrm{~nm}$ in diameter. Collectively, these fibrils form a 3D meshwork with the physical properties of a malleable, dimensionally-stable hydrogel. 
3D hydrogels for suspension of vascular tissue can be prepared from acidic collagen extracts by combining 1 volume of collagen extract, 1/9 volume of 10X Medium 199 (Invitrogen) or 10X DMEM (Sigma-Aldrich), and sufficient $1 \mathrm{X}$ DMEM and FBS to yield a solution with final collagen and FBS concentrations of $1-1.5 \mathrm{mg} / \mathrm{ml}$ and $2.5 \%$, respectively. If necessary, the $\mathrm{pH}$ of the collagen solution is adjusted to $7.0-7.2$ with $0.1 \mathrm{~N} \mathrm{HCl}$ or $\mathrm{NaOH}$. To provide additional buffering capacity, the $10 \mathrm{X}$ culture medium can be supplemented with powdered $\mathrm{NaHCO}_{3}$, added to saturation.

To avoid premature polymerization, it is important to chill all of the components of the collagen solution on ice prior to combining them. Best results are obtained when the acidic collagen extract is added to the mixture last. All components should be combined in a centrifuge tube and mixed slowly by hand, rocking the tube end-over-end 5-8 times. Vigorous mixing should be avoided, as it can cause the collagen to precipitate or polymerize rapidly into clumps of short fibrils, resulting in a mechanically weak, opaque gel. These tendencies are increased in solutions that are excessively basic; therefore, it is important to adjust the $\mathrm{pH}$ to be no greater than 7.2.

\subsection{Fabrication and Culture of 3D Gel Assemblies with Vascular Explants}

The fragility of 3D collagen gels can make analyses involving staining and imaging problematic. We have found that explants of murine microvasculature and aortae can be embedded within low-volume $(30 \mu \mathrm{l})$ collagen gels supported at the edges by rings of nylon mesh. This approach optimizes specimen handling, staining, and economical use of reagents. Importantly, the preparations can be readily imaged by conventional widefield microscopy or confocal microscopy.

Assemblies comprised of nylon mesh rings, 3D collagen gels, and vascular explants are prepared as follows: UVsterilized Nylon (Nitex ${ }^{\mathrm{TM}}$ ) mesh rings (mesh type 03-100/44) with inner and outer diameters of $3 \mathrm{~mm}$ and $5.6 \mathrm{~mm}$, respectively, are placed on the undersides of $60 \mathrm{~mm}$ diameter plastic cell culture dish tops lined with UV-sterilized sheets of Parafilm $\mathrm{M}^{\mathrm{TM}}$ (Pechiney Plastic Packaging) to create a hydrophobic surface. The rings are then flooded with $10 \mu \mathrm{l}$ of collagen solution and the dishes covered with the dish bottoms, which have a disk of moist filter paper pressed into them to provide humidity. The preparations are then placed for 30 minutes in a tissue culture incubator at $37^{\circ} \mathrm{C} / 5 \% \mathrm{CO}_{2} / 100 \%$ humidity to gel the collagen. Subsequently, single aortic segments or 3-5 segments of adipose microvasculature (the latter suspended in a small volume of culture medium) are placed on the collagen gel, carefully overlaid with an additional $20 \mu \mathrm{l}$ of the collagen solution, and returned to the incubator for 30 minutes to gel the overlay. The assemblies are then transferred to 96-well cell culture plates filled with $50 \mu$ (per well) of MCDB 131 or other media supplemented with $0-2.5 \%$ FBS and antibiotics. If desired, 5-20 $\mathrm{ng} / \mathrm{ml}$ of recombinant human VEGF $_{165}$ (Peprotech) can be added to stimulate the outgrowth of sprouts. After 2-10 days of culture (depending on the type of explant), with media changed every 2 days for the longer-term cultures, the assemblies are rinsed in phosphate-buffered saline (PBS), fixed 20 minutes in $10 \%$ neutral-buffered formalin (NBF), and stored in PBS for subsequent analysis.

We have the nylon mesh rings made for us commercially, either by cold-stamping with a sharp-edged punch or by laser-cutting. We prefer cold-stamped rings to laser-cut rings, as the former have clean, incised edges, whereas the latter can have melted edges of greater irregularity. Commercially-made rings are relatively expensive, but provide a highly uniform product and save the time required for fabrication. However, to limit expense, rings can be cut out of bulk nylon mesh (available from McMaster-Carr, Inc.) using disposable $3 \mathrm{~mm}$ and $6 \mathrm{~mm}$ diameter biopsy punches (Thermo Fisher Scientific, Inc.) for the inner and outer diameters, respectively.

\subsection{Analyses of Sprouts Produced in 3D Vascular Cultures}

Microvascular explants (Fig. 1) give rise to multicellular sprouts after 2-4 days of culture. The sprouts can continue to elongate for 2-3 weeks. To facilitate identification of sprouts, particularly during early stages of growth, it is useful to record phase-contrast images of the explants within a few hours after embedment in collagen for comparison with the culture at later times. Typically, sprouts emerge from one or both ends of an explant and not from the middle. Emergent single cells are excluded from analysis. Over time in culture, the main body of the explant can lose its tubular form and assume a rounded or irregular contour. Aortic segments typically give rise to well-defined multicellular sprouts within 7 days in vitro [18] (Fig. 2).

After fixation in NBF, the nuclei within microvascular and aortic sprouts can be labeled with conventional fluorescent nucleic acid dyes such as DAPI, propidium iodide, or TO-PRO ${ }^{\circledR}-3$ iodide and the cell cytoplasm labeled with Alexa-Fluor ${ }^{\mathrm{TM}}$ phalloidin (Molecular Probes/Invitrogen), which binds to f-actin. After labeling, the 3D gel assemblies are placed on slides and coverslipped using a mounting medium that inhibits photobleaching, such as Vecta-Shield ${ }^{\circledR}$ (Vector Laboratories). The nylon mesh rings of the assemblies act as spacers that permit the collagen gels to be flattened slightly between the slide and coverslip, but not crushed. Consequently, the assemblies remain intact and can be de-mounted from the slides for additional washing and/or restaining (several times, if necessary) by immersing the slides on edge in PBS until the coverslips slide off. As an alternative to imaging by fluorescence, fixed sprout preparations can be visualized by staining with a general protein stain, such as $2 \%$ crystal violet for 10 minutes, followed by multiple washes in PBS or TBS until the collagen gels become transparent.

Sprout formation from labeled preparations can be quantified by use of MetaMorph $\AA$ (Molecular Devices) or other image analysis software according to parameters of cell number, lumen diameter, and branching morphology. For sprouts from microvascular explants, we found that the length of new vascular outgrowths between branch points is not significantly affected by various growth conditions and that differences in growth between preparations can be quantified simply by counting the number of branch points in each sprout [15]. Morphological measurements of microvascular and aortic sprouts can be made from images 

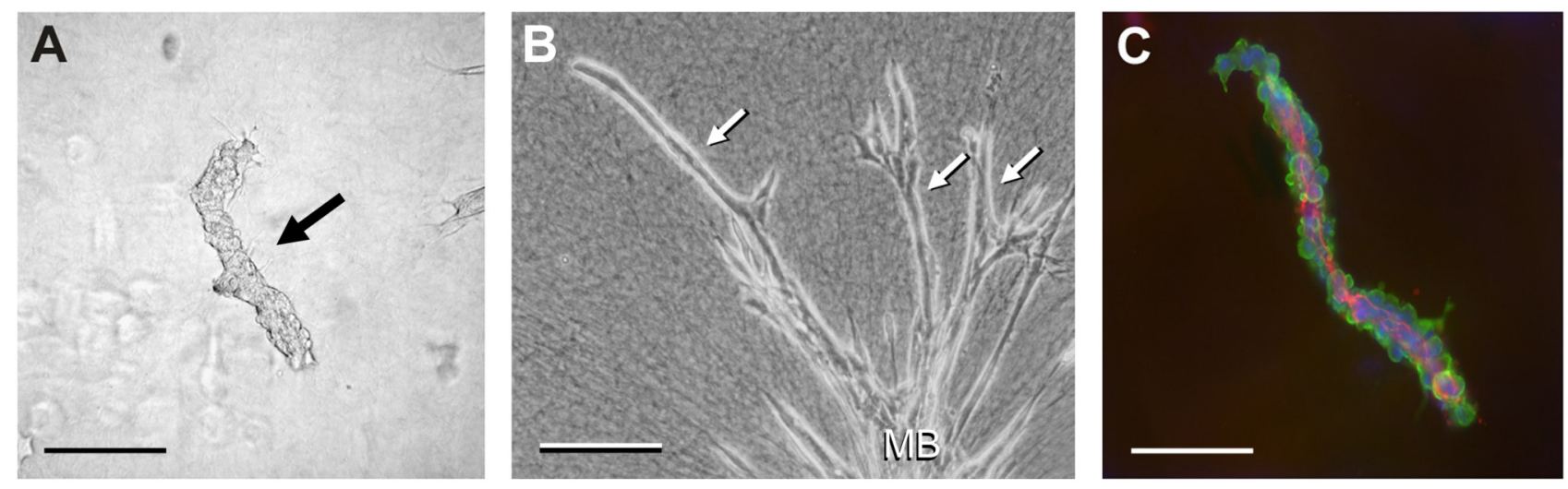

Fig. (1). Murine microvascular segments suspended in 3D collagen gels. A) A freshly-isolated microvascular segment (arrow). B) A similar segment has produced long sprouts (three are labeled with arrows) from its main body (MB) after 14 days of culture. C) A segment labeled with antibodies to PECAM (red) to identify ECs of the endothelial lining, antibodies to alpha smooth muscle actin (green) to reveal supportive mural cells, and DAPI (blue) to show the cell nuclei. Scale bars in A and C $=100 \mu \mathrm{m}$. Scale bar in B $=200 \mu \mathrm{m}$.

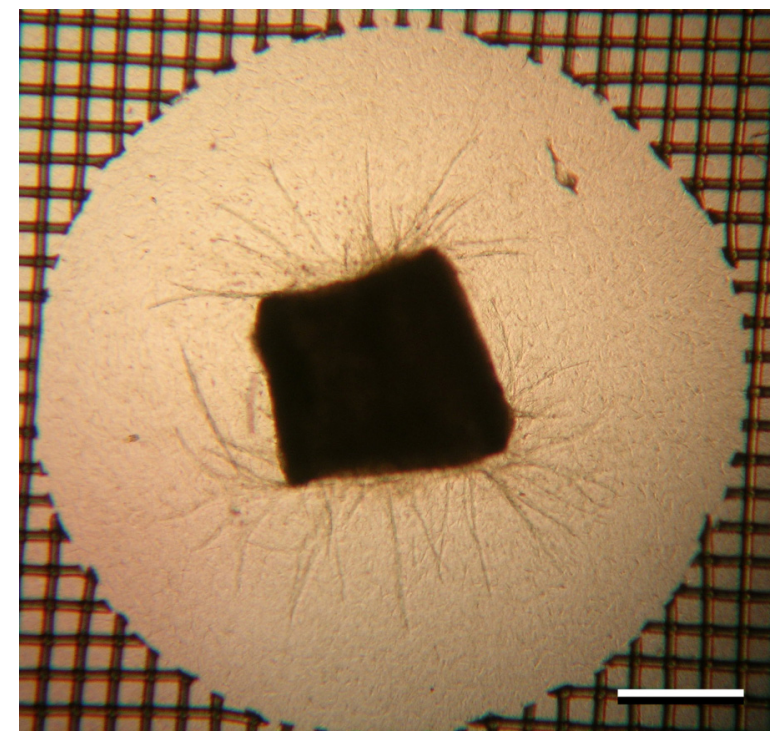

Fig. (2). A murine aortic segment cultured in a 3D collagen gel for 7 days has produced a large number of sprouts. A portion of the supportive ring of nylon mesh can be seen at the periphery of the image. Scale bar $=500 \mu \mathrm{m}$.

taken by conventional wide field microscopy; however, greater discrimination, which includes $3 \mathrm{D}$ reconstruction of Z-stacked images, can be achieved with a confocal microscope. ECs can be distinguished from mural cells by live cell uptake of fluorescent 1,1'-dioctadecyl - 3,3,3',3'tetramethyl-indocarbocyanine perchlorate (DiI)-labeled acetylated low-density lipoprotein (Biomedical Technologies, Inc.) or by indirect immunofluorescence (IIF) with anti-CD31/PECAM antibodies. Mural cells can be identified by their negativity for EC markers or by positive immunofluoresence for alpha smooth muscle actin or desmin. IIF staining procedures for EC and mural cell markers, or other target molecules, will vary with the antibodies used. Typically, we enhance the permeability of the sprout preparations by a 15-30 minute exposure to PBS $/ 0.01 \%$ Triton ${ }^{\circledR} \mathrm{X}-100$, followed by a $1-2$ hour block in $\mathrm{PBS} / 2 \%$ normal serum from the species in which the secondary antibody is raised. The blocked preparations are exposed for 3-18 hours to primary antibodies at concentrations between $10-50 \mu \mathrm{g} / \mathrm{ml}$ in blocking solution, followed by a 2-3 hour wash in PBS and a 1-2 hour exposure to Alexa-Fluor-tagged secondary antibodies at 5$10 \mu \mathrm{g} / \mathrm{ml}$ in blocking solution. Subsequently, the labeled preparations are washed for at least 1 hour. Should background staining be excessive, as revealed by fluorescence microscopy, the preparations can be washed for an additional period (overnight, if necessary) until backgrounds are acceptable.

\subsection{Additional Considerations}

Successful isolation of microvascular segments from epididymal fat involves a number of variables, which include the degree of maceration of the isolated fat, the activity of the type II collagenase, and the time, temperature and degree of agitation during digestion. Microvessels will not be released from under-digested fat, whereas over-digestion will yield fragile microvessels that will subsequently disintegrate. Establishing the conditions that optimize the yield of viable microvessels requires a certain amount of trial and error.

Isolation of aortic segments is technically easier than isolation of microvascular segments from fat; however, 
aortic segments can include fibroblasts, which can migrate from the explant into the surrounding collagen gel and interfere with the angiogenic response. Fibroblast contamination can be decreased substantially by carefully removing the loose perivascular connective tissue from the aorta prior to cutting it into segments. Removal of the connective tissue can be performed by a combination of two techniques, which involve: 1) holding the aorta at one end with a relatively coarse-tipped watchmaker's tweezer (e.g., a \#1) and pulling the tags of connective tissue away with a fine-tipped tweezer (e.g., a \#3 or \#5) held in the opposite hand, and 2) holding the aorta at one end with the coarsetipped tweezer and using a small dissecting scissors to trim the connective tissue away. Scissors with the blades set at an angle to the handle are particularly useful, as the angle allows the blades to be brought parallel to the aorta for a close cut that removes superficial tissue without penetrating the aortic wall. After the aorta is cleaned of connective tissue, the portion of the aorta held by the coarse tweezer (which is usually crushed) can be trimmed away and discarded.

\section{SUMMARY}

In vitro models of angiogenesis within 3D ECM permit effective visualization and measurement of vascular growth. Models utilizing isolated, dispersed ECs are relatively easy to set up and can generate multicellular tubes with a high degree of similarity to native capillaries; however, such models may not reflect true angiogenesis in that parent blood vessels are absent, at least during the initial formation of tubes. Alternative methods to concentrate isolated ECs in aggregates or on microcarriers that act as parent vessel-like foci for directional sprouting into surrounding ECM have certain limitations, but continue to be useful.

As a complementary approach to the use of isolated ECs, segments of explanted microvessels or aortae can act as parent blood vessels for angiogenesis-like sprout formation in vitro. These models can be used to evaluate a multiplicity of factors that regulate sprouting that include: 1) the composition and physical characteristics of the surrounding ECM, and 2) exogenously-added drugs, biological agents, or other chemical compounds with the potential to promote or inhibit angiogenesis. These parameters can be evaluated in either limited-sample formats or in larger, high-throughput assays. For larger-scale analyses, the miniaturized version of the explant model we described allows an efficient utilization of cells and reagents which may be expensive or in limited supply.

The lenticular collagen gels formed by the methods outlined in this report have optical properties that are favorable for imaging by conventional and confocal microscopy. Furthermore, the mechanical support provided by the nylon mesh rings facilitates handling and staining of the gels with dyes and antibodies. Consequently, techniques of image analysis can be used to quantify sprout formation according to morphological parameters of cell number, lumen diameter, and degree of branching, as well as to analyze the spatial and temporal expression of gene products identified by specific cytochemical labels.

As a final consideration, the capacity of explant models to use vasculature from mice allows the investigator to study vascular growth using the full spectrum of genetic tools developed for this species, which includes various genetic knock-out and knock-in strategies, as well as genes engineered for transcriptional regulation by specific compounds, such as doxycycline. Moreover, expression of specific genes by sprouting ECs or mural cells can be directly visualized by use of fluorescent reporters under control of target promoters.

\section{ACKNOWLEDGEMENTS}

We acknowledge the following grant support: NIH grants R01 AG015837 and R21 AG024458 [MJR] and R21 EB005652 [RBV].

\section{REFERENCES}

[1] Davis, G.E.; Camarillo, C.W. An alpha 2 beta 1 integrin-dependent pinocytic mechanism involving intracellular vacuole formation and coalescence regulates capillary lumen and tube formation in threedimensional collagen matrix. Exp. Cell Res., 1996, 224, 39-51.

[2] Koike, T.; Vernon, R.B.; Gooden, M.D.; Sadoun, E.; Reed, M.J Inhibited angiogenesis in aging: a role for TIMP-2. J. Gerontol. A Biol. Sci. Med. Sci., 2003, 58, B798-805.

[3] Vernon, R.B.; Sage, E.H. A novel, quantitative model for study of endothelial cell migration and sprout formation within threedimensional collagen matrices. Microvasc. Res., 1999, 57, 118-133.

[4] Nehls, V.; Drenckhahn, D. A novel, microcarrier-based in vitro assay for rapid and reliable quantification of three-dimensional cell migration and angiogenesis. Microvasc. Res., 1995, 50, 311-322.

[5] Wagner, R.C.; Matthews, M.A. The isolation and culture of capillary endothelium from epididymal fat. Microvasc. Res., 1975 , 10, 286-297.

[6] Nicosia, R.F.; Tchao, R.; Leighton. J. Histotypic angiogenesis in vitro: light microscopic, ultrastructural, and radioautographic studies. In vitro, 1982, 18, 538-549.

[7] Sato, N.; Sawasaki, Y.; Senoo, A.; Fuse, Y.; Hirano, Y.; Goto, T. Development of capillary networks from rat microvascular fragments in vitro: the role of myofibroblastic cells. Microvasc. Res., 1987, 33, 194-210.

[8] Mori, M.; Sadahira, Y.; Kawasaki, S.; Hayashi, T.; Notohara, K.; Awai. M. Capillary growth from reversed rat aortic segments cultured in collagen gel. Acta Pathol. Jpn., 1988, 38, 1503-1512.

[9] Diglio, C.A.; Grammas, P.; Giacomelli, F.; Wiener, J. Angiogenesis in rat aorta ring explant cultures. Lab. Invest., 1989, $60,523-531$.

[10] Nicosia, R.F.; Ottinetti, A. Growth of microvessels in serum-free matrix culture of rat aorta. A quantitative assay of angiogenesis in vitro. Lab. Invest., 1990, 63, 115-122.

[11] Nicosia, R.F.; Bonanno, E.; Villaschi. S. Large-vessel endothelium switches to a microvascular phenotype during angiogenesis in collagen gel culture of rat aorta. Atherosclerosis, 1992, 95, 191199.

[12] Hoying, J.B.; Boswell, C.A.; Williams, S.K. Angiogenic potential of microvessel fragments established in three-dimensional collagen gels. In vitro Cell. Dev. Biol. Anim., 1996, 32, 409-419.

[13] Zhu, W.H.; Nicosia, R.F. The thin prep rat aortic ring assay: a modified method for the characterization of angiogenesis in whole mounts. Angiogenesis, 2002, 5, 81-86.

[14] Krishnan, L.; Hoying, J.B.; Nguyen, H.; Song, H.; Weiss, J.A. Interaction of angiogenic microvessels with the extracellular matrix. Am. J. Physiol. Heart Circ. Physiol., 2007, 293, H36503658 .

[15] Arthur, W.T.; Vernon, R.B.; Sage, E.H.; Reed, M.J. Growth factors reverse the impaired sprouting of microvessels from aged mice. Microvasc. Res., 1998, 55, 260-270.

[16] Masson, V.V.; Devy, L.; Grignet-Debrus, C.; Bernt, S.; Bajou, K.; Blache, S.; Roland, G.; Chang, Y.; Fong, T.; Carmeliet, P.; Foidart, J.M.; Noe, A. Mouse aortic ring assay: a new approach of the molecular genetics of angiogenesis. Biol. Proce. Online, 2002, 4, 24-31.

[17] Zhu, W.H.; Iurlaro, M.; MacIntyre, A.; Fogel, E.; Nicosia, R.F. The mouse aorta model: influence of genetic background and aging on bFGF- and VEGF-induced angiogenic sprouting. Angiogenesis, 2003, 6, 193-199. 
[18] Reed, M.J.; Karres, N.; Eyman, D.; Vernon, R.B. Culture of murine aortic explants in 3-dimensional extracellular matrix: A novel, miniaturized assay of angiogenesis in vitro. Microvasc. Res., 2007, $73,248-252$.

[19] Nunes, S.S.; Greer, K.A.; Stiening, C.M.; Chen, H.Y.; Kidd, K.R.; Schwartz, M.A.; Sullivan, C.J.; Rekapally, H.; Hoying, J.B. Implanted microvessels progress through distinct neovascularizaion phenotypes. Microvasc. Res., 2010, 79, 10-20.

[20] Yang, X.; Staren, E.D.; Howard, J.M.; Iwamura, T.; Bartsch, J.E.; Appert, H.E. Invasiveness and MMP expression in pancreatic carcinoma. J. Surg. Res., 2001, 98, 33-39.

[21] Chandrakasan, G.; Torchia, D.A.; Piez, K.A. Preparation of intact monomeric collagen from rat tail tendon and skin and the structure of the nonhelical ends in solution. J. Biol. Chem., 1976, 251, 60626067.

[22] Rajan, N.; Habermehl, J.; Cote, M.F.; Doillon, C.J.; Mantovani. D. Preparation of ready-to- use, storable and reconstituted type I collagen from rat tail tendon for tissue engineering applications. Nat. Protoc., 2006, 1, 2753-2758.

[23] Damodarasamy, M.; Vernon, R.B.; Karres, N.; Chang, C.H.; Bianchi-Frias, D.; Nelson, P.S.; Reed, M.J. Collagen extracts derived from young and aged mice demonstrate different structural properties and cellular effects in three-dimensional gels. $J$. Gerontol. A Biol. Sci. Med. Sci., 2010, 65, 209-218.

(C) Reed et al.; Licensee Bentham Open.

This is an open access article licensed under the terms of the Creative Commons Attribution Non-Commercial License (http://creativecommons.org/licenses/by-nc/3.0/) which permits unrestricted, non-commercial use, distribution and reproduction in any medium, provided the work is properly cited. 